\title{
Optimization of Airport Surface Traffic: A Case-study of Incheon International Airport
}

\author{
8 June 2017
}

Yeonju Eun*, Daekeun Jeon,

Myeongsook Jeong, Hyounkyong Kim,

Eunmi Oh, and Sungkwon Hong,

Korea Aerospace Research Institute
Hanbong Lee, Yoon Jung NASA Ames Research Center

Zhifan Zhu

SGT, NASAAmes Research Center
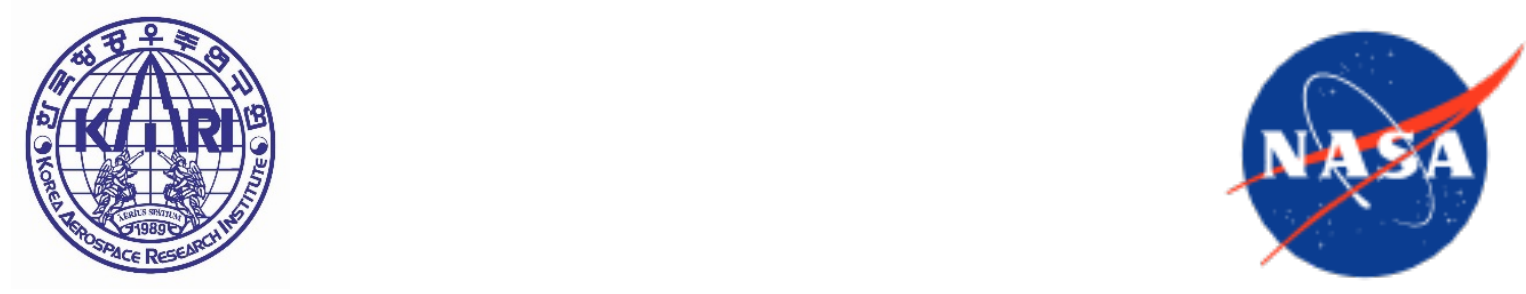


\title{
Contents
}

\author{
Introduction
}

Scheduling Requirements

Runway Scheduling

Taxiway Scheduling

Optimization Test

Conclusion 


\section{Introduction}

- Incheon International Airport (ICN) in South Korea

- Surface congestion due to continuously growing traffic demands

- Airport expansion project in progress

- Growing need for CDM and controller decision support tool

\section{- Research Purpose}

- SW Development of a decision support tool for IADS (Integrated Arrival, Departure, Surface) operation in ICN

- Research collaboration between Korea Aerospace Research Institute (KARI) and National Aeronautics and Space Administration (NASA)

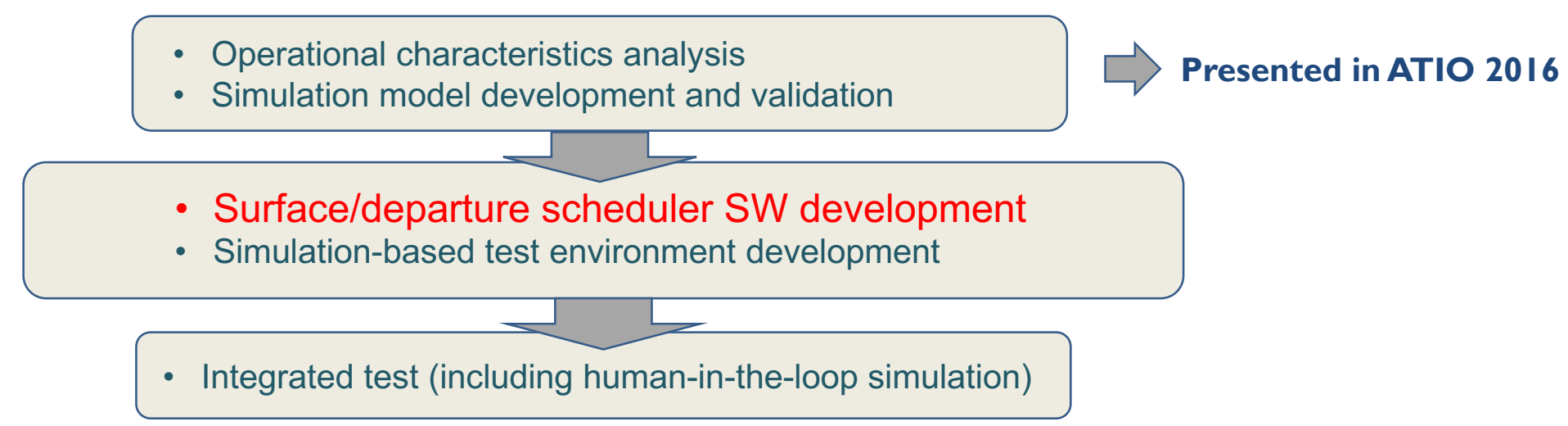




\section{Introduction}

- Research Direction

- Based on 3-step approach
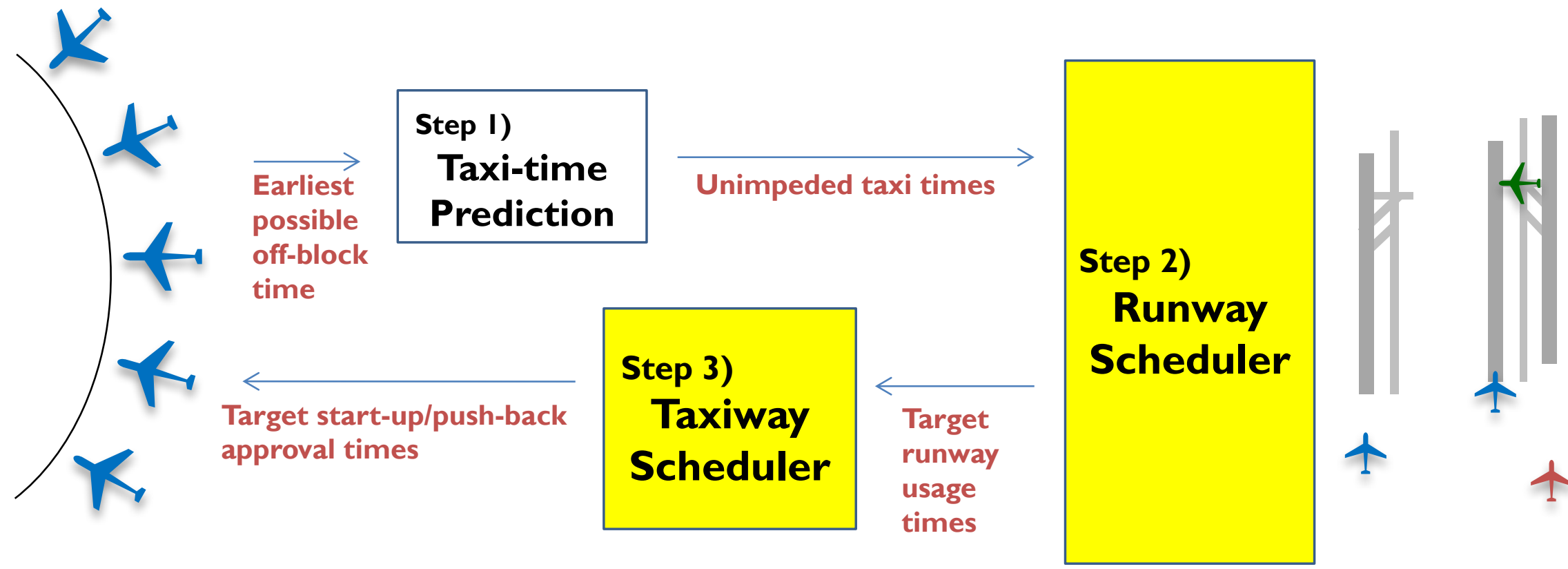

- MILP-based optimization models were developed and tested. 


\section{Scheduling Requirements}

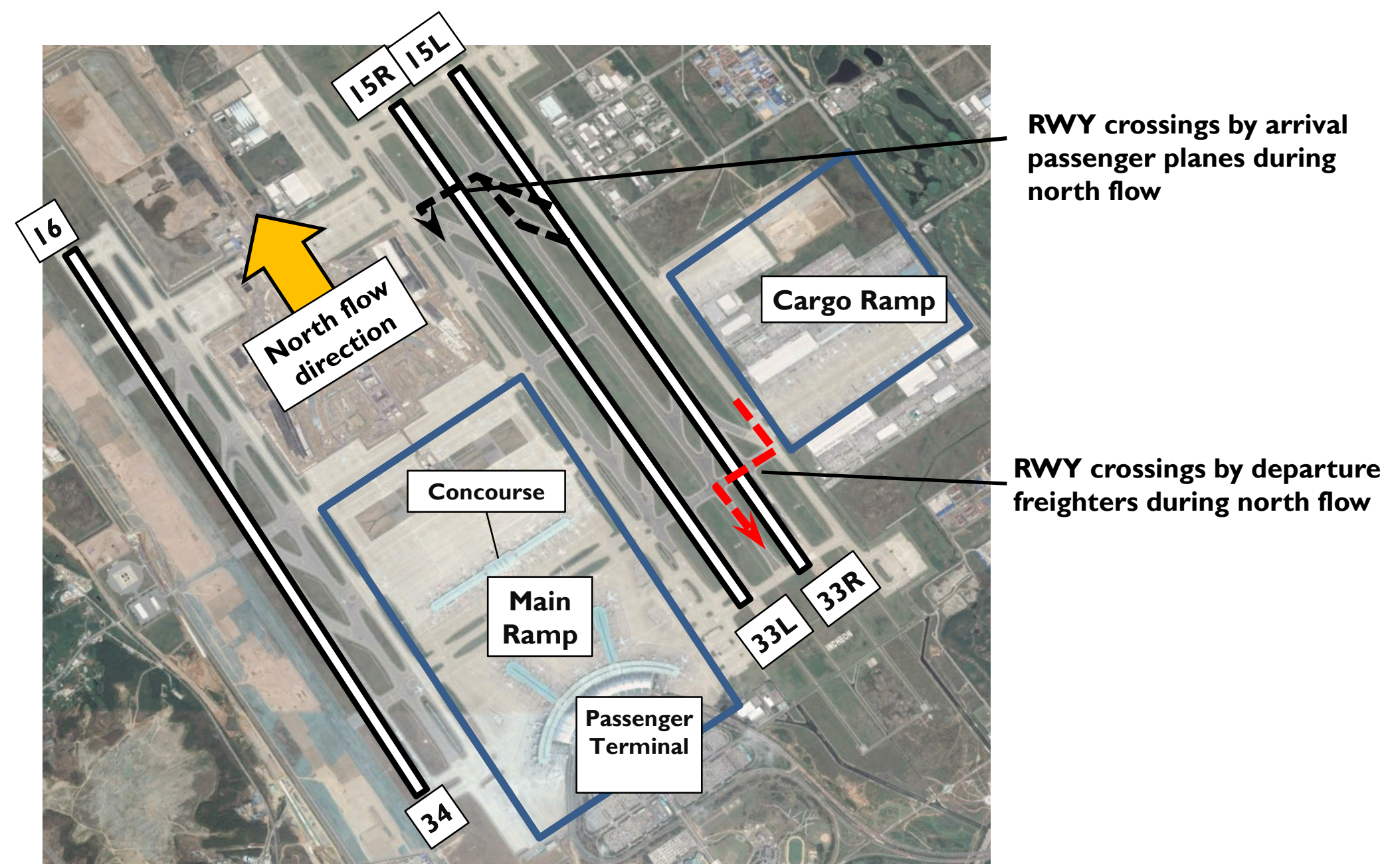

\section{Airport Configuration}




\section{Scheduling Requirements}
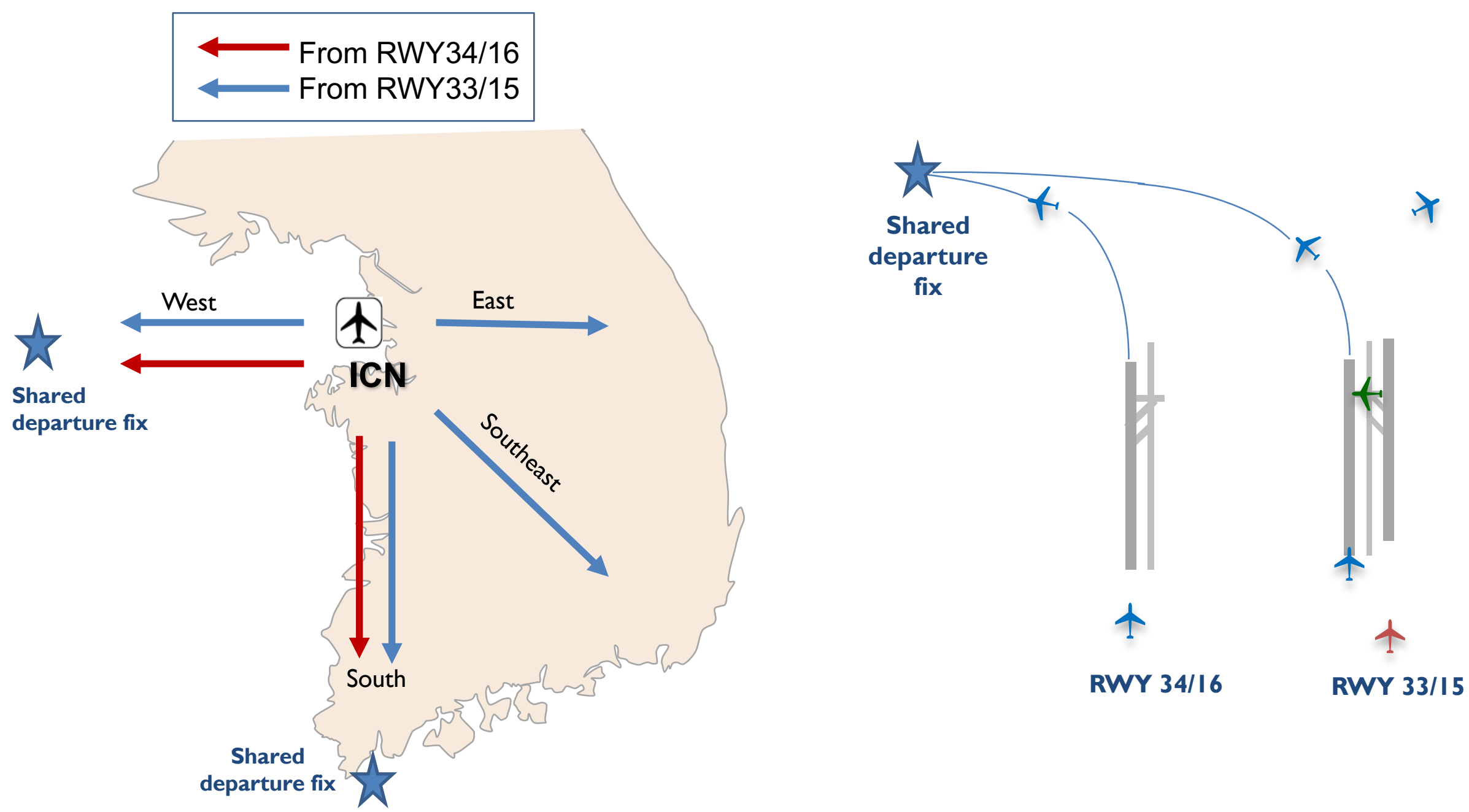

Departure route directions and a shared departure fix from the multiple runways in ICN 


\section{Scheduling Requirements}

\section{Multiple Take-off Time Windows}

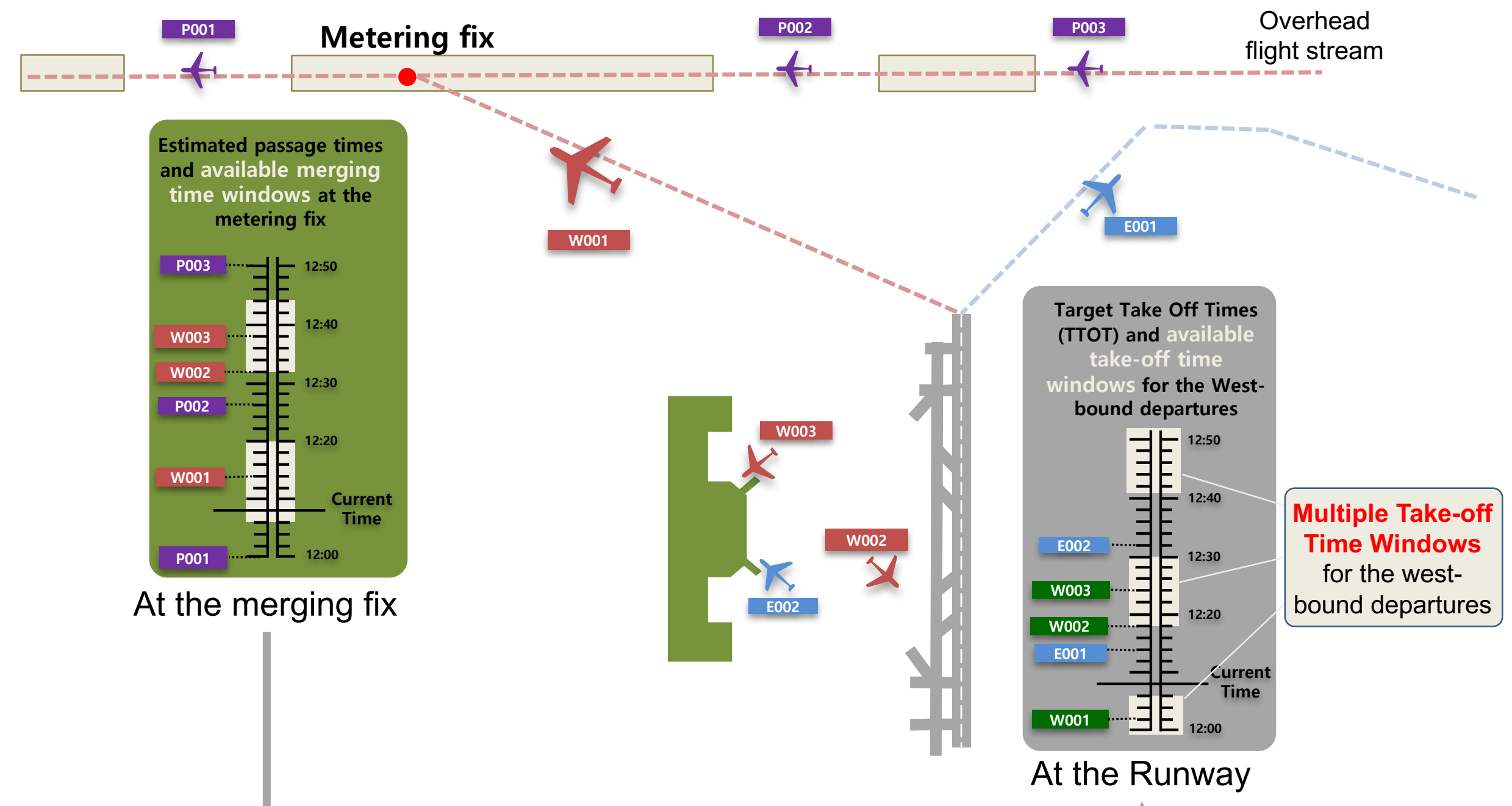




\section{Scheduling Requirements}

- Multiple runway scheduling

- With shared departure fixes

- TMls (Traffic Management Initiatives)

- CFR

- EDCT

- MIT/MDI

- Multiple takeoff time windows

- Runway crossings

- Departure runway crossings by arrival flights

- Arrival runway crossings by departure freighters

- Gate holding and pushback time limit

- Earliest and/or latest takeoff time limit

- ELDT (Expected Landing Time)

- Assumed to be given and not adjustable

- Taxi route of each aircraft

- Assumed to be given and not adjustable
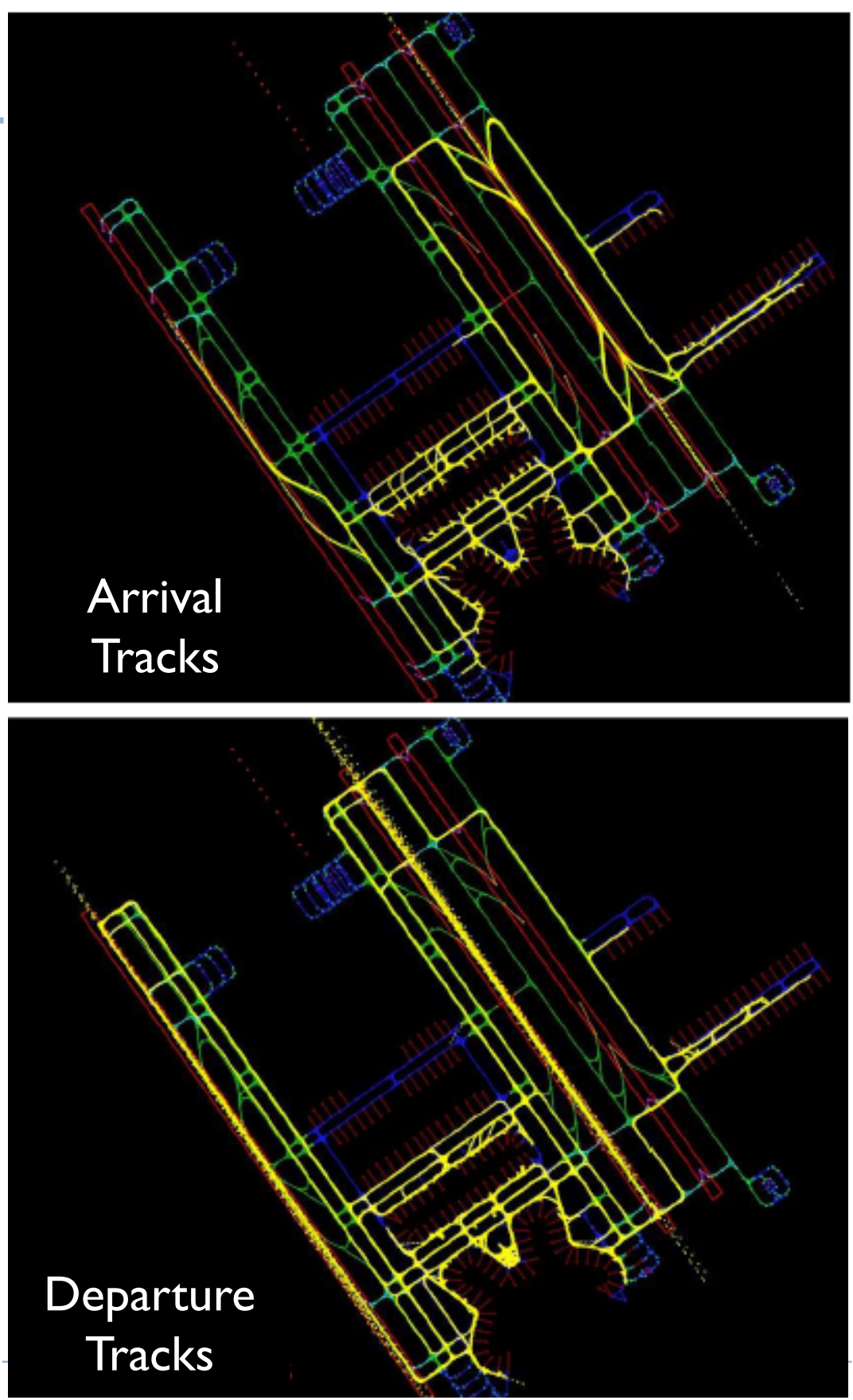


\section{Runway Scheduling}

minimize $\sum_{i \in D}\left(t_{i}-\right.$ Earliest $\left._{i}\right)$

subject to $z_{i j}+z_{j i}=1, \quad \forall i, j \in D \bigcup A \bigcup C, i \neq j$

$t_{j}-t_{i}-\operatorname{Rsep}_{i j} \geq-M\left(1-z_{i j}\right), \quad \forall i, j \in D \cup A \cup C, i \neq j$

Earliest $_{i} \leq t_{i} \leq$ Latest $_{i}, \quad \forall i, j \in D \cup A \cup C, i \neq j$

$z_{i j} \in\{0,1\}, \forall i, j \in D \cup A \cup C$

$z_{i j}=1, \quad \forall i, j \in D_{\text {Class }_{k}}, \quad$ Earliest $_{i}<$ Earliest $_{j}, \quad i \neq j$

\section{For ICN RWY scheduler,}

$\forall i \in D, \quad\left\{\begin{array}{l}\text { Earliest }_{i}=\text { EarliestOff }_{i} \\ \text { Latest }_{i}=\text { EarliestOffT }_{i}+\text { MaxRunwayDelay }_{i}\end{array}\right.$

$\forall i \in A, \quad$ Earliest $_{i}=$ Latest $_{i}=\mathrm{OnT}_{i}$

$\forall i \in C,\left\{\begin{array}{l}\text { EarliestT }_{i}=\mathrm{OnT}_{i}+\mathrm{TransT}_{i} \\ \text { Latest }_{i}=\min \left\{\mathrm{OnT}_{j}+\mathrm{TransT}_{j} \mid \forall j \in C: \mathrm{OnT}_{i}<\mathrm{OnT}_{j}\right\}\end{array}\right.$ 


\section{Runway Scheduling}

\section{<Additional Constraints for TMls>}

- EDCT, CFR $\rightarrow$ Adjustment of EarliestT $T_{i}$ and Latest $T_{i}$

- MIT(Miles-In-Trail), MDI (Minimum Departure Interval)

$$
\begin{aligned}
& \text { In case of MIT) } \quad t_{j}-t_{i}+\left(\operatorname{TansT}_{j}^{k}-\operatorname{TransT}_{j}^{k}-\frac{\mathrm{MIT}_{k}}{\operatorname{Trans}_{i}^{k}}\right) \geq-M\left(1-z_{i j}\right), \forall i, j \in D_{M I T_{k}}, i \neq j \\
& \text { In case of MDI) } t_{j}-t_{i}+\left(\mathrm{MDI}_{k}\right) \geq-M\left(1-z_{i j}\right), \forall i, j \in D_{M D I_{k}}, i \neq j
\end{aligned}
$$

- Multiple Take-off Time Windows

Time $_{i}=\left\{\left\lfloor\right.\right.$ MinTime $_{i, 1}$, MaxTime $\left._{i, 1}\right\rfloor\left\lfloor\right.$ MinTime $_{i, 2}$, MaxTime $\left._{i, 2}\right\rfloor, \cdots, \mid$ MinTime $_{i, N_{W_{i}}}$, MaxTime $\left.\left._{i, N_{W_{i}}}\right\rfloor\right\}$

$$
\begin{aligned}
& s_{i}^{k}= \begin{cases}1 & \text { if } \text { MinTime }_{i, k} \leq t_{i} \leq \text { MaxTime }_{i, k} \\
0 & \text { otherwise }\end{cases} \\
& s_{i}^{k} \in\{1,0\}, \quad \forall i \in D_{\text {TimeW }}, k \in\left(1 . . N_{W_{i}}\right), \quad \sum_{k=1}^{N_{W_{i}}} s_{i}^{k}=1, \quad \forall i \in D_{\text {TimeW }}
\end{aligned}
$$




\section{Taxiway Scheduling}

$\operatorname{minimize} \alpha_{p} \underbrace{\left(\sum_{i \in D, r \in R} \max \left[t_{i, r}-\text { DesiredOffT }_{i, r}, 0\right]\right)}_{\text {Late Take-off Time }}$

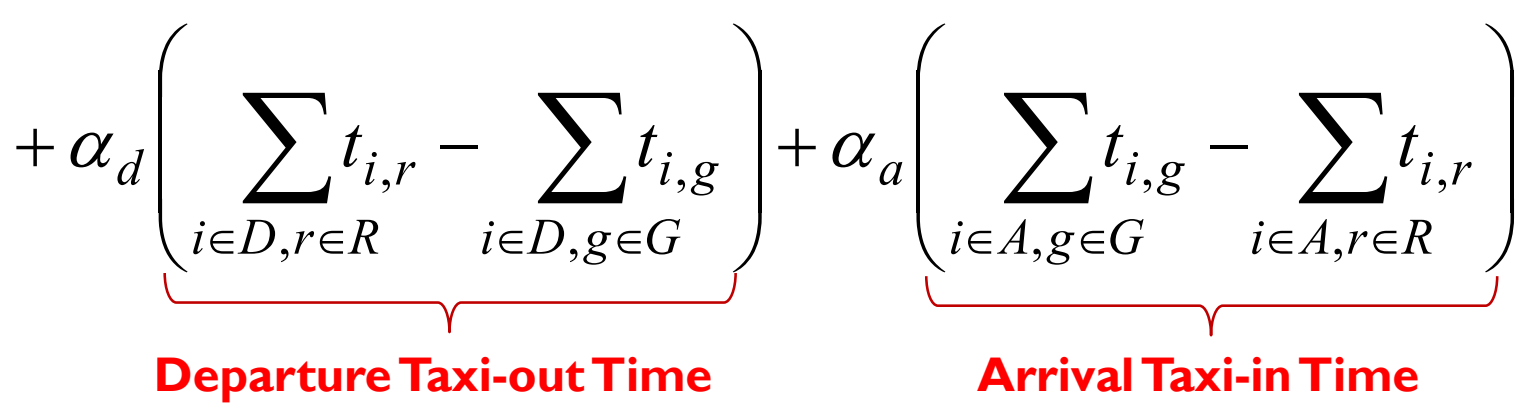

subject to $\quad z_{i j}^{u} \in\{0,1\}, \forall i, j \in D \bigcup A, i \neq j, u \in I \quad$ Passage sequence of flight $\mathrm{i}$ and $\mathrm{j}$ at node u $t_{i, u} \geq 0, \quad \forall i \in D \cup A, u \in N \quad$ Passage time of flight i at node u $z_{i j}^{u}+z_{j i}^{u}=1, \quad \forall i, j \in D \bigcup A, \quad i \neq j, u \in I \quad$ Passage sequence at node u

$t_{i, v} \geq t_{i, u}+\operatorname{MinTaxiT}_{u v}, \quad \forall i \in D \bigcup A,(u, v) \in E \quad$ Minimum travel time in link u-v $z_{i j}^{u}=z_{i j}^{v}, \quad \forall i, j \in D \bigcup A, \quad i \neq j, u, v \in I, \quad(u, v) \in E \quad$ No overtaking allowed along taxiways $z_{i j}^{u}+z_{j i}^{v}=1, \quad \forall i, j \in D \bigcup A, \quad i \neq j, u, v \in I, \quad(u, v) \in E \quad$ Conflict free in bi-directional link 


\section{Taxiway Scheduling}

subject to (continued)

$$
\begin{aligned}
& t_{j, u}-t_{i, u}-\left(t_{i, v}-t_{i, u}\right) \frac{\operatorname{Dsep}_{i j}}{l_{u v}} \geq-\left(1-z_{i j}^{u}\right) M, \quad \forall i, j \in D \bigcup A, \quad i \neq j, u \in I, \quad(u, v) \in E \\
& t_{j, v}-t_{i, v}-\left(t_{j, v}-t_{j, u}\right) \frac{\operatorname{Dsep}_{i j}}{l_{u v}} \geq-\left(1-z_{i j}^{v}\right) M, \quad \forall i, j \in D \bigcup A, i \neq j, \quad v \in I, \quad(u, v) \in E
\end{aligned}
$$

Maintaining required separations at intersections

$t_{j, r}-t_{i, r}-\operatorname{Rsep}_{i j} \geq-\left(1-z_{i j}^{r}\right) M, \quad \forall i, j \in D, \quad i \neq j, \quad r \in R \quad$ Runway separation

$t_{i, r} \geq$ EarliestOffT $_{i, r}, \quad \forall i \in D, r \in R \quad$ Earliest take-off time

$t_{i, g} \geq \mathrm{OutT}_{i, g}, \quad \forall i \in D, g \in G \quad$ Pushback ready time

$t_{i, g} \leq \operatorname{OutT}_{i, g}+$ MaxGateHold $_{i, g}, \quad \forall i \in D, g \in G \quad$ Maximum gate holding time

$t_{i, r}=\mathrm{OnT}_{i, r}, \quad \forall i \in A, \quad r \in R$

Arrival landing time

$t_{i, u}=$ FrozenT $_{i, u}, \quad \forall i \in D^{\prime} \bigcup A^{\prime}, u \in N \quad$ Frozen schedule 


\section{Taxiway Scheduling}

\section{<Additional Constraints for RWY crossings >}

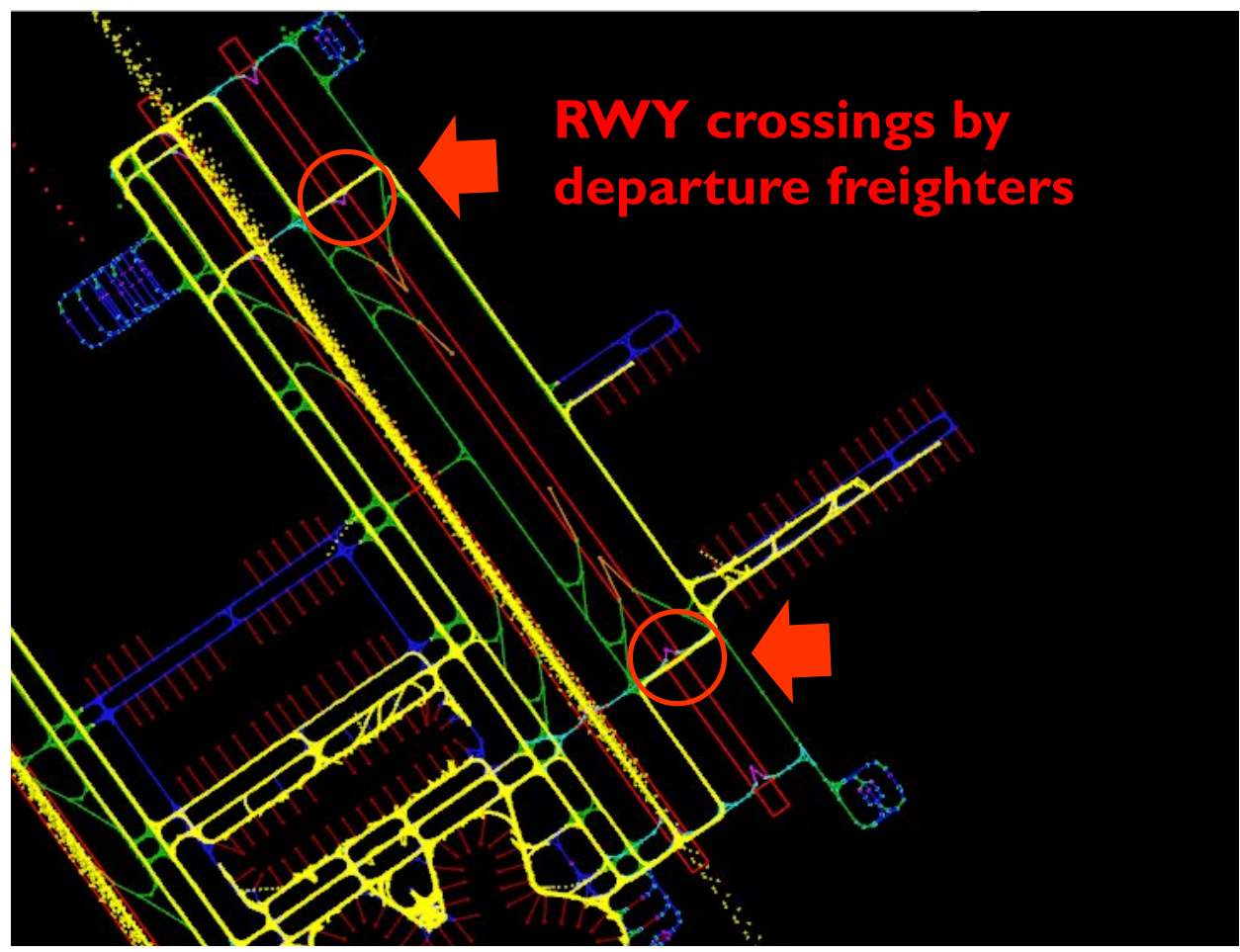

Departure Tracks
$C_{d e p}:$ Set of departure freighters

(which need to cross the arrival runway.)

$$
C_{\text {dep }} \subset D
$$

crossing sequence $=$ departure sequence

$z_{i j}^{c}=z_{i j}^{r} \quad \forall i, j \in C_{d e p}, i \neq j, \quad r \in R$

Runway separation with Arrivals

$$
\begin{aligned}
& t_{j, r}-t_{i, c}-\operatorname{Rsep}_{i j} \geq-M\left(1-z_{i j}^{c r s}\right) \\
& t_{i, c}-t_{j, r}-\operatorname{Rsep}_{j i} \geq-M \cdot z_{i j}^{c r s} \\
& z_{i j}^{c r s} \in\{0,1\}, \quad \forall(i, j) \in\left(C_{d e p} \times A\right)
\end{aligned}
$$




\section{Taxiway Scheduling}

\section{ICN Node-link model for taxiway scheduling}

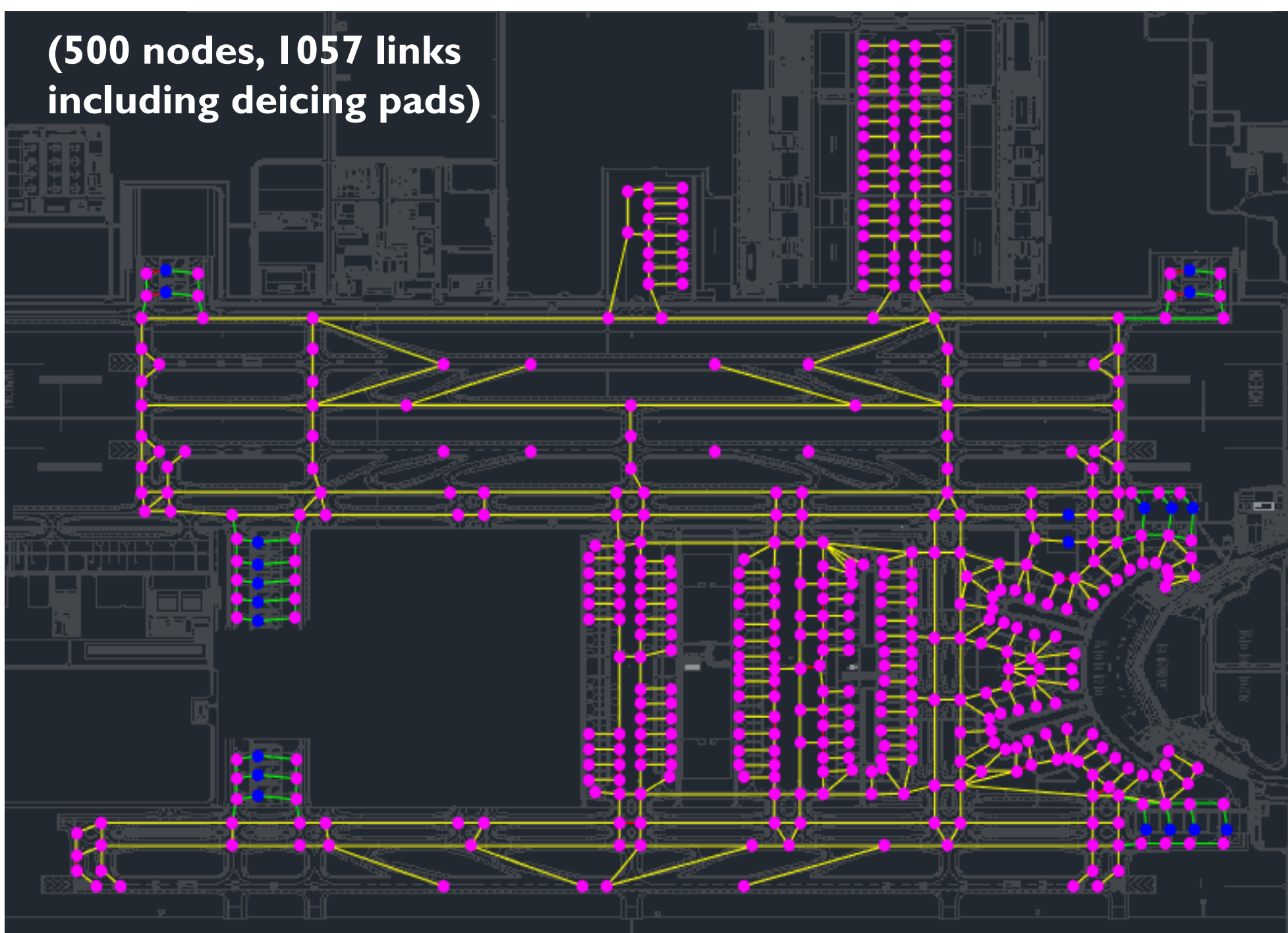




\section{Optimization Tests}

\section{RWY separation matrix}

Separation between Dep and Dep (sec)

\begin{tabular}{|c|c|c|c|c|c|}
\cline { 3 - 6 } \multicolumn{2}{c|}{} & \multicolumn{4}{|c|}{ Tailing Aircraft } \\
\cline { 2 - 6 } \multicolumn{2}{c|}{} & $\mathrm{L}$ & $\mathrm{M}$ & $\mathrm{H}$ & $\mathrm{S}$ \\
\hline \multirow{3}{*}{$\begin{array}{c}\text { Leading } \\
\text { aircraft }\end{array}$} & $\mathrm{L}$ & 120 & 120 & 120 & 120 \\
\cline { 2 - 6 } & $\mathrm{M}$ & 180 & 120 & 120 & 120 \\
\cline { 2 - 6 } & $\mathrm{H}$ & 180 & 180 & 120 & 120 \\
\cline { 2 - 6 } & $\mathrm{S}$ & 180 & 180 & 120 & 120 \\
\hline
\end{tabular}

\begin{tabular}{|c|c|c|c|c|}
\hline \multirow{2}{*}{ Dep } & L & M & H & S \\
\cline { 2 - 5 } & 80 & 52 & 45 & 45 \\
\hline
\end{tabular}

\begin{tabular}{|c|c|c|c|c|}
\hline \multirow{2}{*}{ Arr } & L & M & H & S \\
\cline { 2 - 5 } & 85 & 47 & 40 & 40 \\
\hline
\end{tabular}

\begin{tabular}{|c|c|c|c|c|}
\hline \multirow{2}{*}{ Crs } & $L$ & $M$ & $H$ & $S$ \\
\cline { 2 - 5 } & 30 & 30 & 30 & 30 \\
\hline
\end{tabular}

RWY occupancy times (sec)

Separation between Dep and Arr : RWY occupancy time of a preceding aircraft +10 sec Separation between Dep and Crs : RWY occupancy time of a preceding aircraft +10 sec

Separation between operations on independent RWYs : Osec 


\section{Optimization Tests}

- Single Scenario Test

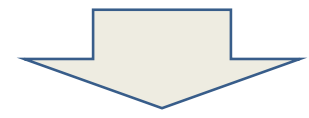

- Purpose) Optimization results check for both runway scheduling and taxiway scheduling.

- Test Scenario) Based on the real operation data of April 2015, the number of departures was assumed to be increased by $30 \%$ from a normal traffic volume.
- Monte-Carlo Test

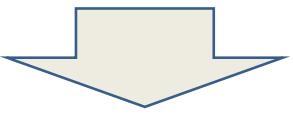

- Purpose) Computation time performance check for the multiple runway scheduling problem.

- Test Scenario) Number of departures and arrivals are assumed to be same with the current peak time operation. For each test case, 100 randomly generated scenarios were used. 


\section{Optimization Tests - single scenario test}

\section{Scenario)}

48 departures +12 arrivals during 09:00-10:00

- 19 departures + 12 arrivals + 9 crossings on RWY33/15

- 29 departures on RWY34/16

- 4 departures from RWY33/15 and 11 departures from RWY34/16 merge into same route (South-bound)

\begin{tabular}{|c|c|c|c|c|c|c|c|c|c|}
\hline \multirow{2}{*}{$\begin{array}{c}12 \\
\text { Arrivals }\end{array}$} & \multirow{2}{*}{$\begin{array}{l}12 \text { on } \\
\text { RWY33/15 }\end{array}$} & L & M & $\mathrm{H}$ & $S$ & \multirow{2}{*}{\multicolumn{4}{|c|}{9 PAX(RWY crossing accompanied)+ 3 CGO }} \\
\hline & & & 3 & 9 & & & & & \\
\hline \multirow{3}{*}{$\begin{array}{c}48 \\
\text { Departures }\end{array}$} & \multirow{2}{*}{$\begin{array}{l}19 \text { on } \\
\text { RWY33/15 }\end{array}$} & & \multirow{2}{*}{5} & \multirow{2}{*}{13} & \multirow{2}{*}{1} & W-bound & S-bound & SE-bound & E-bound \\
\hline & & & & & & 0 & 4 & 8 & 7 \\
\hline & $\begin{array}{l}29 \text { on } \\
\text { RWY34/16 }\end{array}$ & & 13 & 16 & & 18 & 11 & 0 & 0 \\
\hline
\end{tabular}

\section{Constraints)}

CPS : 3

TMI : MIT on West-bound/South-bound 


\section{Optimization Tests - single scenario test}

From RWY33/15, $\mathrm{H}$ or $\mathrm{SH}$

- - - - · From RWY33/15, L or M
From RWY34/16, $\mathrm{H}$ or SH

- - - - · From RWY34/16, L or M

No MIT

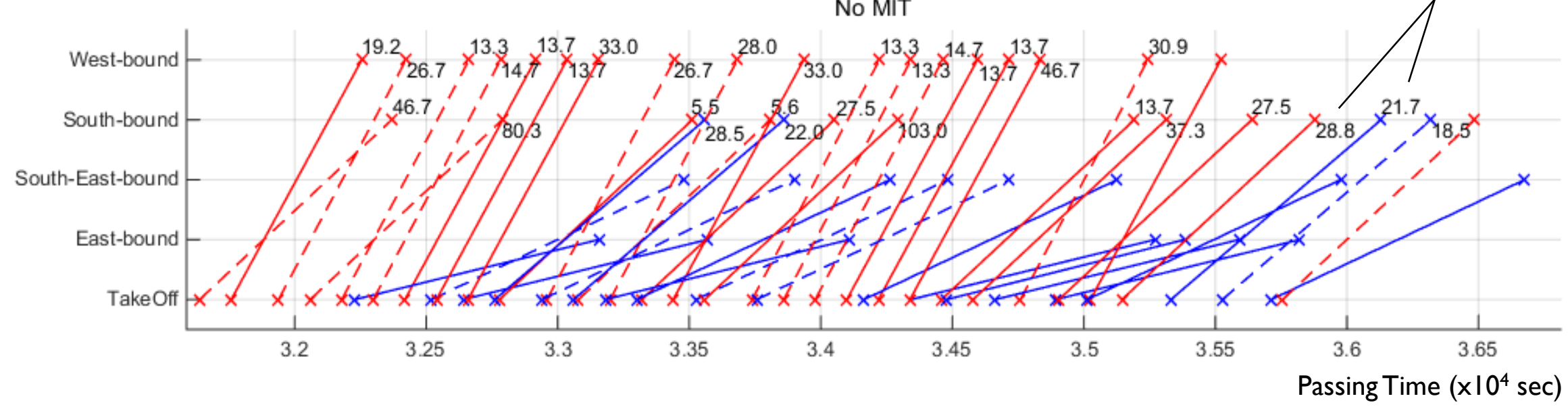

15NM-MIT on West and South-bound

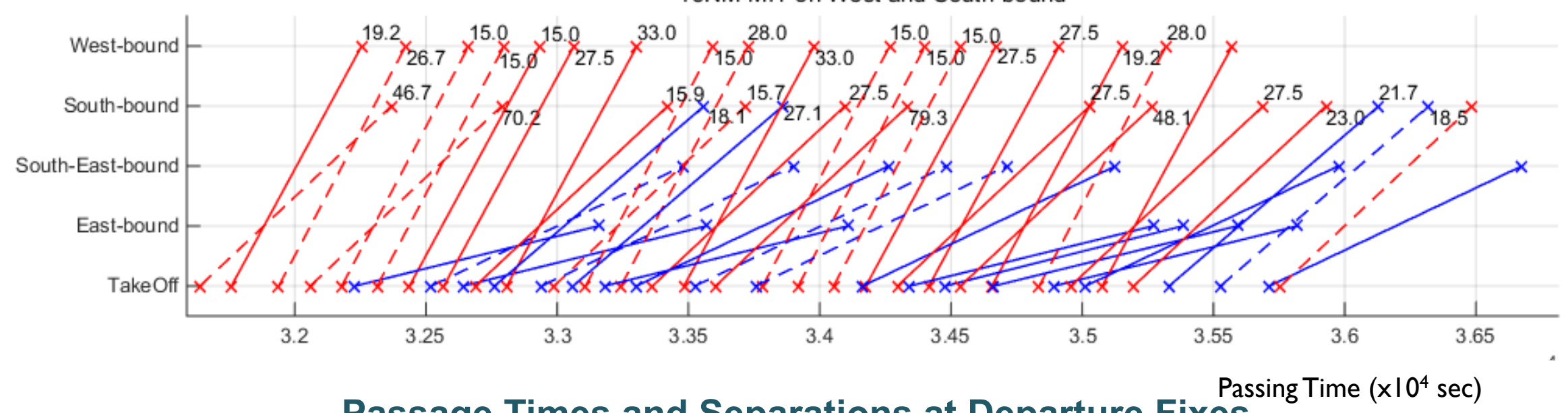

Passage Times and Separations at Departure Fixes 


\section{Optimization Tests - single scenario test}
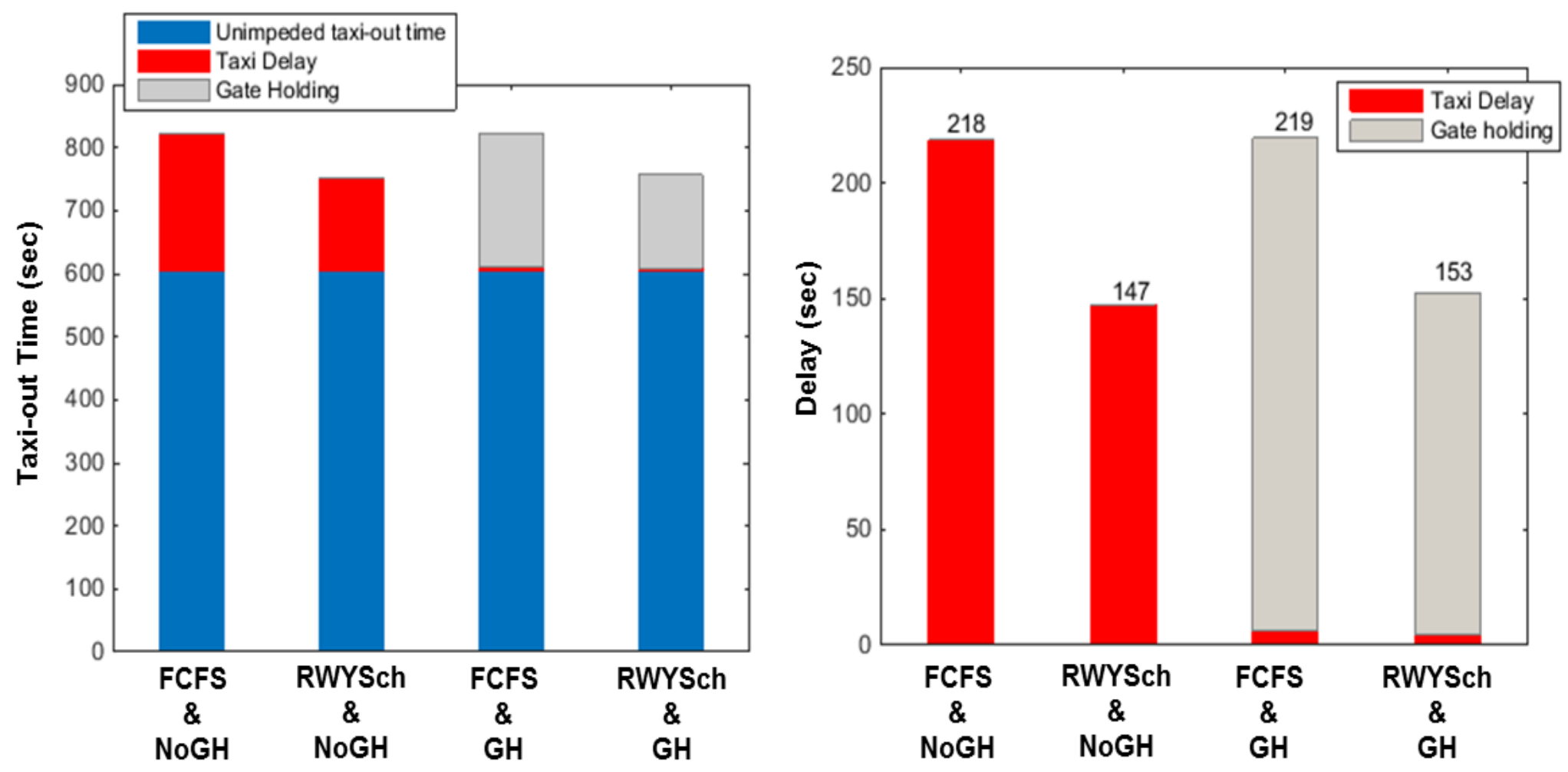

Averaged taxi-out time and delay per departure aircraft 


\section{Optimization Tests - Monte-Carlo test}

\section{Two different methods for the multiple runway scheduling problem}

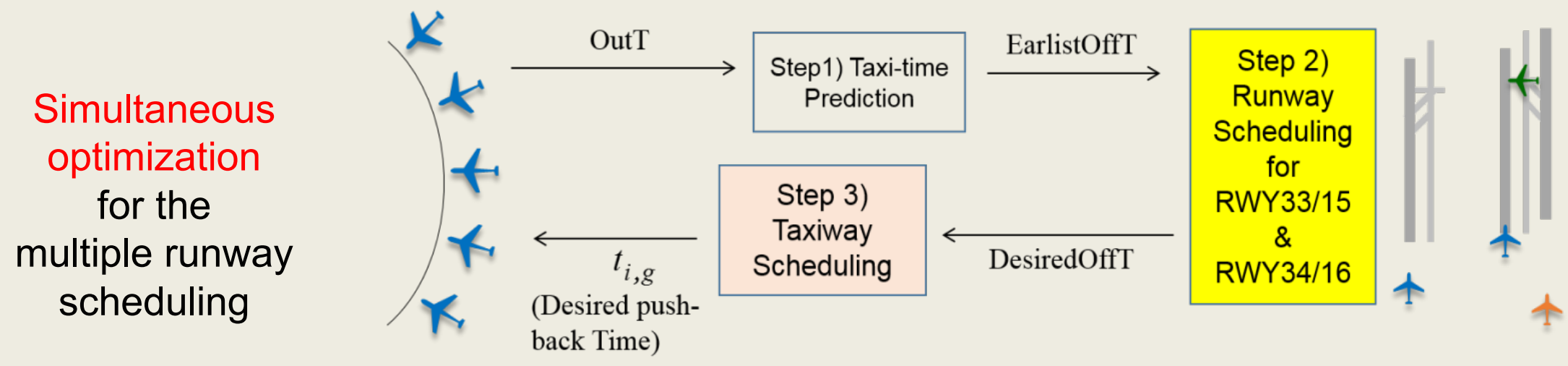

Sequential optimization

for the multiple runway scheduling

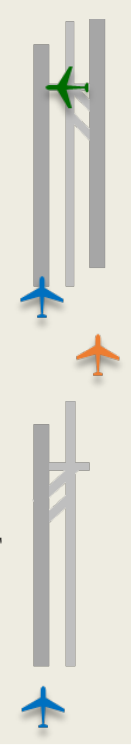




\section{Optimization Tests - Monte-Carlo test}

\section{Test scenarios}

- 40 departures + 20 arrivals for 1 hour (the number of departure runways: 2 )

- 15NM MIT separation on south-bound departures

- Involves all south-bound departures from both runways to the shared departure fix.

- 100 random scenarios for each test case

\begin{tabular}{|c|c|c|}
\hline & \multicolumn{2}{|c|}{ The total number of departures $=40$} \\
\hline from RWY 33L/I5R (to the shared fix) & from RWY $34 / 16$ (to the shared fix) \\
\hline Case 0 & $15(5)$ & $25(10)$ \\
\hline Case I & $14(4)$ & $26(11)$ \\
\hline Case 2 & $13(3)$ & $27(12)$ \\
\hline Case 3 & $12(2)$ & $28(13)$ \\
\hline Case 4 & $11(1)$ & $29(14)$ \\
\hline Case 5 & $10(0)$ & $30(15)$ \\
\hline
\end{tabular}

- The total number of the south-bound departures to the shared departure fix are same.

- The south-bound departures which take-off from RWY 33L/I5R were re-assigned to RWY34/16 one-by-one over case $0-5$. 


\section{Optimization Tests - Monte-Carlo test}

Test results: computation time comparison

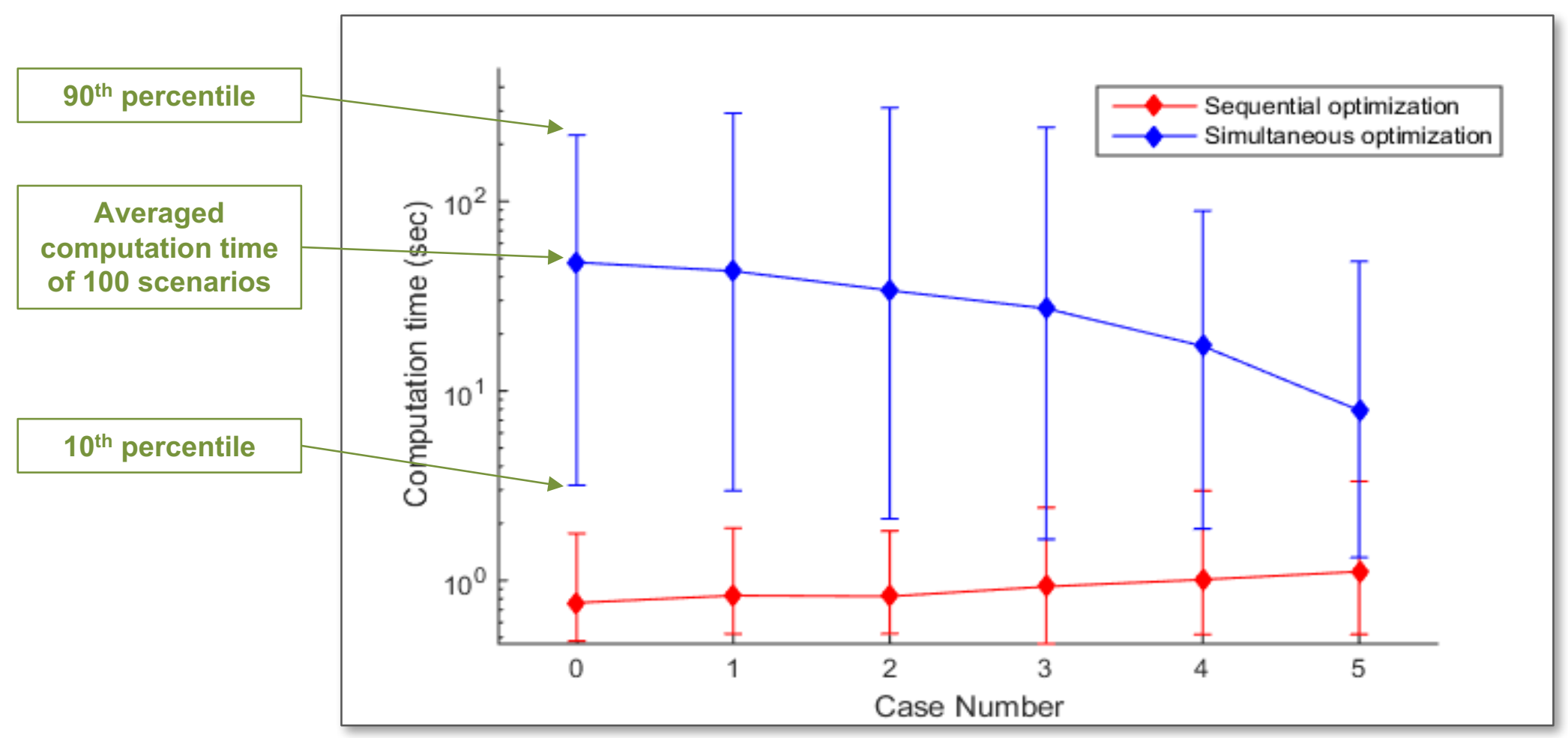

Computation time comparison in a log scale 


\section{Optimization Tests - Monte-Carlo test}

\section{Test results: Optimization cost comparison}

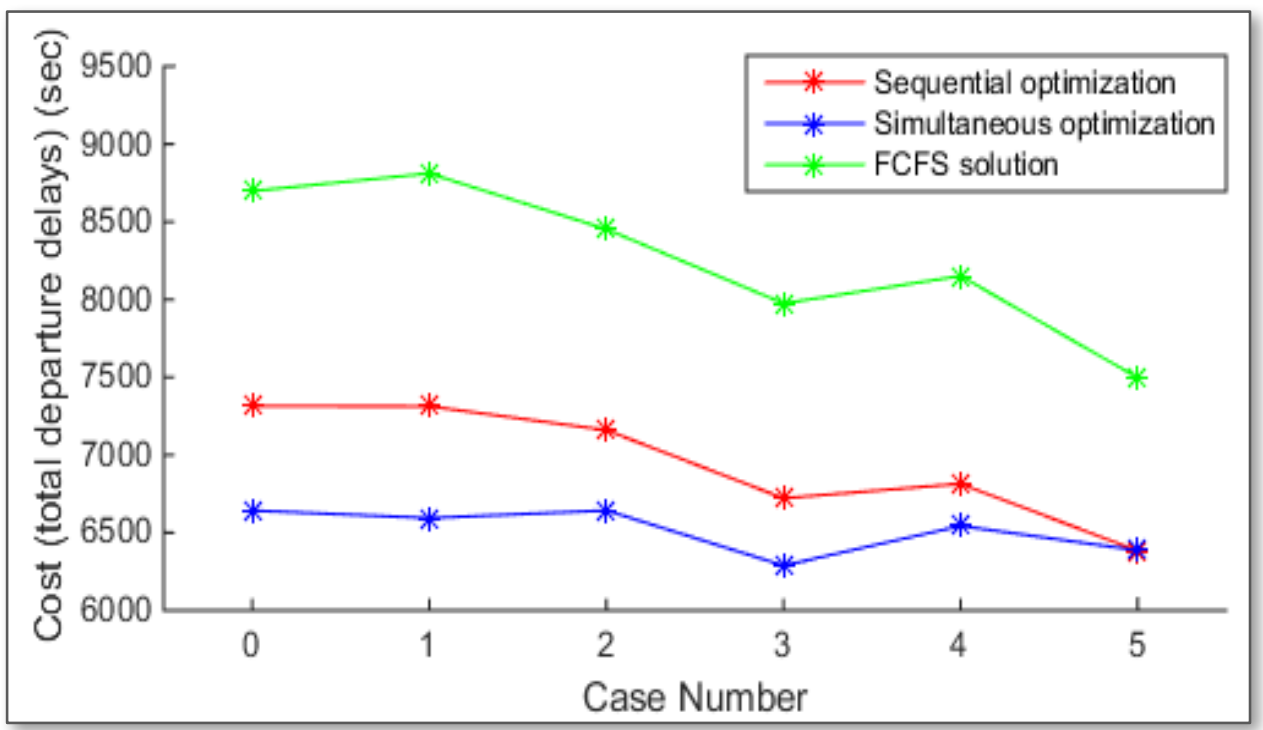

Optimization cost comparison

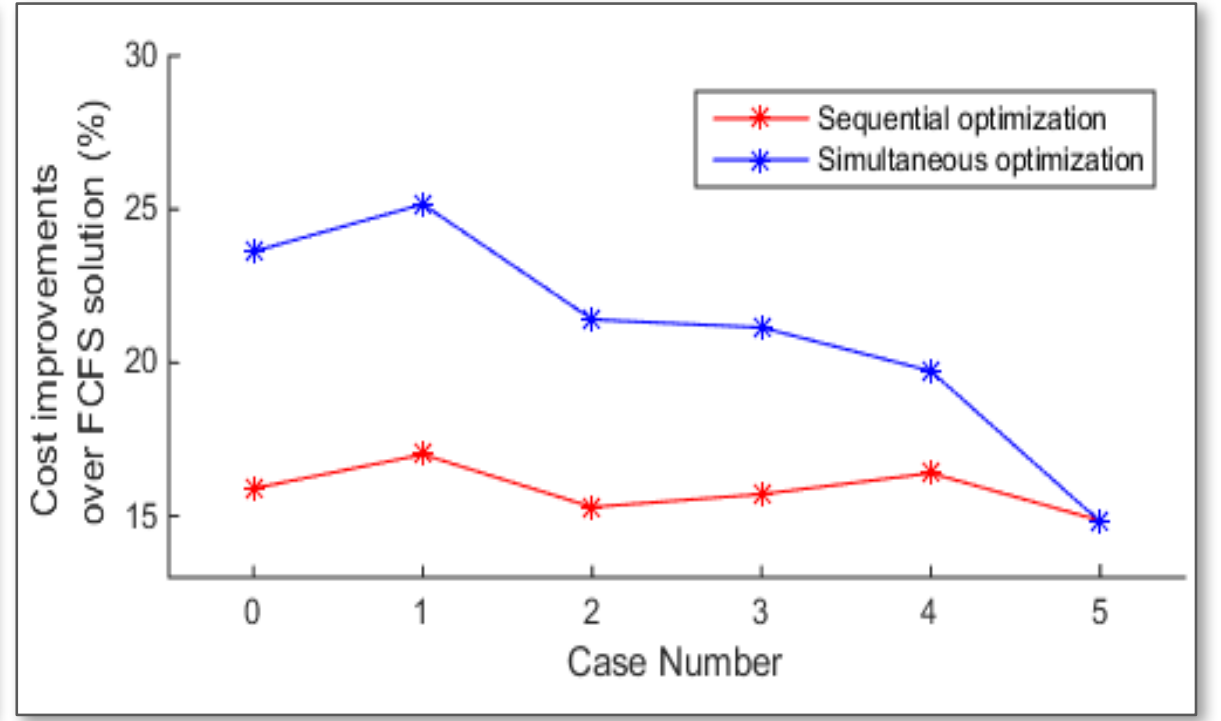

Cost improvements over FCFS solution 


\section{Conclusion}

Developed the optimization models for airport surface traffic scheduling

- MILP-based optimization models for runway scheduling and taxiway scheduling were developed and tested.

- TMls and operational characteristics which are specific to ICN were incorporated.

- Multiple runway scheduling with consideration for MIT(Miles-In-Trail) separation at the shared departure fix

v 'Multiple take-off time windows' constraints

v Two different types of runway crossings on the coupled runways 33L/15R and 33R/15L.

\section{- Suggested a method for better computation time performance}

- The sequential optimization using 'multiple take-off time windows' was proposed.

- The sequential optimization shows much better performance with reasonably low cost for the multiple runway scheduling problem.

- Future Works

- Integration of the additional requirements from ANSP (Air Navigation Service Provider) of ICN, such as cruise altitude assignment to the departure flights in pre-departure sequencing stage.

- Runway assignment problem for runway balancing at an airport with multiple departure runways. 


\section{ThankYYou}

Contact to: yjeun@kari.re.kr 